\title{
Overview of microgrid systems
}

\author{
V. Saravanan ${ }^{1}$, K. M. Venkatachalam ${ }^{2}$, M. Arumugam ${ }^{3}$, M. A. K. Borelessa ${ }^{4}$, K. T. M. U. Hemapala \\ ${ }_{1,2,3}$ Department of Electrical \& Electronics Engineering, Arunai Engineering College, Tamil Nadu, India \\ ${ }^{4,5}$ Department of Electrical Engineering, University of Moratuwa, Moratuwa, Sri Lanka
}

\begin{tabular}{|c|c|}
\hline Article Info & ABSTRACT \\
\hline Article history: & This research paper discusses the different types of microgrids, their \\
\hline Received Oct 19, 2020 & structural arrangements and the technology adopted for different power \\
\hline Revised Jun 21, 2021 & security plans used for optimal performance. A detailed overview of the \\
\hline Accepted Oct 14, 2021 & $\begin{array}{l}\text { direct current (DC) microgrid system is discussed, outlining its } \\
\text { configurations and technical-economic aspects. Performance evaluation of }\end{array}$ \\
\hline Sovwords. & microgrid carried out through various reliability codes is also provided. \\
\hline
\end{tabular}

Control \& protection

Microgrid

Power management

Schematic arrangement

This is an open access article under the CC BY-SA license.

Corresponding Author:

V. Saravanan

Department of Electrical and Electronics Engineering

Arunai Engineering College

Tiruvannamalai 606 603, Tamilnadu, India

Email: vsaranaec@yahoo.co.in

\section{INTRODUCTION}

The microgrid is an electrical power system that consists of distributed renewable energy sources energy storage systems and loads, which can be operated either in grid-connected or isolated/stand-alone modes. This section deals with the definition, components, characteristics, benefits, and necessity of microgrid (MG). A microgrid, a part of the distribution system, with its power generation sources and loads can form an isolated electric power system. During normal operating conditions, MG can be connected to the alternating current (AC) grid at the point of common coupling (PCC), and the loads are supplied from the local sources and/or from the ac grid. If the load power requirement is less than the power produced by the available local sources such as solar photovoltaics (PV) arrays, fuel cells, and microturbines, excess power can be exported to the AC grid. These power sources produce power with different voltage amplitude and frequency, therefore, need to be interfaced to the grid/load through power electronic converters [1]-[3].

The microgrid, as defined by the U.S. Department of Energy, is "a group of interconnected loads and distributed energy resources (DERs) with clearly defined electrical boundaries that acts as a single controllable entity for the grid and can connect and disconnect from the grid to enable it to operate in both grid-connected or island modes". The microgrid can be operated in two modes, either grid-connected or islanded. In grid-connected mode, MG trades power with the utility grid, whereas in the islanded mode, MG operates autonomously without connection to the utility grid. DER installations could be considered as an MG when it has clear electrical boundaries, can supply critical load, and is operated by an appropriate controller as a single entity. The major components of MG include DERs, power converters, energy storage, loads, master controller, smart switches, protective devices, as well as communication, control, and 
automation systems. MG protection system consists of fuses, circuit breakers, protective relays, measurement equipment, and grounding.

MG can be categorized into three classes, such as AC microgrid, direct current (DC) microgrid, and hybrid AC/DC microgrid. Among the three, the AC microgrid is popular, because it has a plug-in approach for all DERs and it needs additional power conversion devices. DC microgrid is most suitable for DC loads such as light-emitting diode (LED)/liquid crystal display (LCD), communication/computing devices, variable speed drives and it requires minimum power conversion devices. A hybrid microgrid integrates both $\mathrm{AC}$ and DC subgrids. MG loads are categorized into fixed and flexible loads. Fixed loads cannot be altered but curtailed/deferred, while flexible loads are responsive to control signals. MG can be categorized based on the type (university campus, defense, residential, commercial, and industrial, island, remote villages, emergencies, refugee camps, remote mining operations), size (small, medium, and large scales), the application (premium power, resilience-oriented, and loss reduction), and the connectivity (remote and gridconnected). MGs are low or medium voltage grids located at or near the consumption sites, which can generate power from renewable sources and with energy storage systems that can be included to offer real and reactive power support.

MG offers benefits for customers/utility grid as a whole to improve reliability and resiliency to the local distribution network, to maintain high power quality with reduced carbon emissions, and enhance economic operation by reducing transmission and distribution ( $\mathrm{T} \& \mathrm{D}$ ) costs and effective and energyefficient utilization of renewable sources in response to the real-time market prices. Table 1 gives the benefits of the microgrid.

Table 1. Benefits of microgrid

\begin{tabular}{ll}
\hline \multicolumn{1}{c}{ Off-grid microgrids } & \multicolumn{1}{c}{ Grid-connected microgrids } \\
\hline Secure access to local energy & Secure access to local or grid energy \\
Minimize fossil fuel dependence & Resilience from using multiple sources \\
Maximize the integration of renewables & Energy flexibility through arbitrage savings \\
Reduced energy cost and emissions & Reduced energy cost and emissions \\
\hline
\end{tabular}

\section{ARRANGEMENT AND POWER MANAGEMENT SCHEMES OF HYBRID AC/DC MICROGRID STRUCTURES}

Hybrid AC/DC microgrid contains both AC/DC power sources and AC/DC loads. Based on the connections of the sources and loads, hybrid AC/DC microgrids can be classified into AC-coupled, DCcoupled, and AC-DC-coupled microgrids and their descriptions are given follows. The power management strategies of these hybrid MG determines the output active and reactive powers of these DGs and storage elements and control of their voltages and frequency [4].

\subsection{AC-coupled hybrid microgrid}

The arrangement of AC-coupled hybrid microgrid is shown in Figure 1, where various distributed generation (DGs) and storage elements (SEs) are connected to the common AC bus through their interfacing converters. SE employs bidirectional converters to ensure bidirectional power flow capability. This structure is commonly used, when RE sources produce grid-level AC voltages directly or indirectly through interfacing power converters.

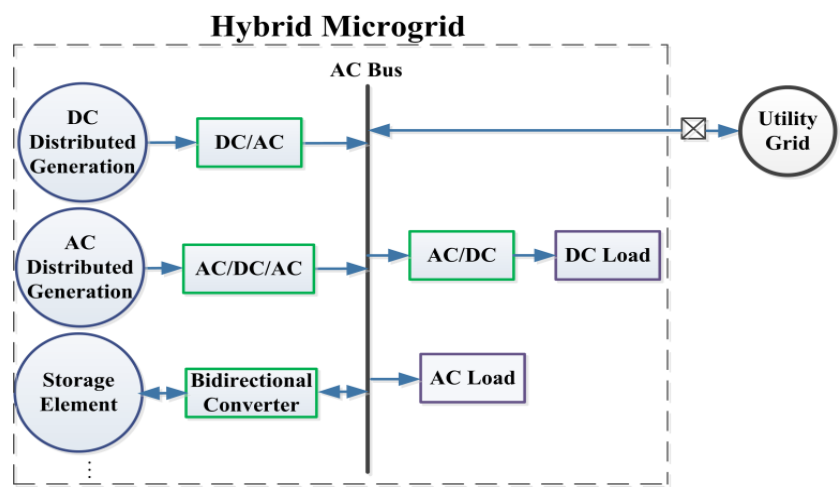

Figure 1. Arrangement of AC-coupled hybrid microgrid 
Control strategies and power management (PM) schemes of AC-coupled hybrid MG are mainly focused on power balancing within the $\mathrm{MG}, \mathrm{AC}$ bus voltage and frequency control in stand-alone operation mode. The overview of power management schemes for AC-coupled MG is shown in Figure 2 which can be separated into grid-connected and stand-alone operation modes.

In grid-connected mode, PM strategies can be classified into dispatched power mode (where the power exchange between the MG and the main grid is dispatched from a higher-level control/optimization scheme) and undispatched output power mode (where the MG output power is not dispatched). In standalone operation mode, PM schemes are focused on MG AC bus voltage and frequency control, as well as demand power-sharing among DGs and SEs.

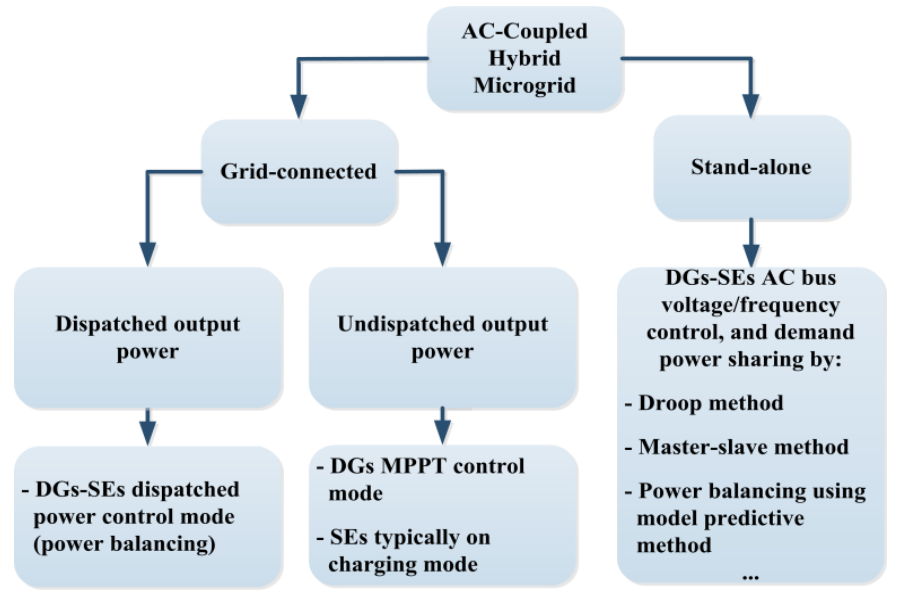

Figure 2. Power management strategies of AC-coupled hybrid microgrid

\subsection{DC-coupled hybrid microgrid}

Figure 3 shows DC-coupled hybrid MG, where DGs and SEs are connected to the common DC bus, and interfacing converters (IFCs) are used to link the DC/AC buses. This structure is good when DC power sources are major power generation units in the MG. In this system, IFCs provide bidirectional power flow between $\mathrm{AC}$ and DC buses and DC-coupled MG does not need any synchronization when integrating different DGs.

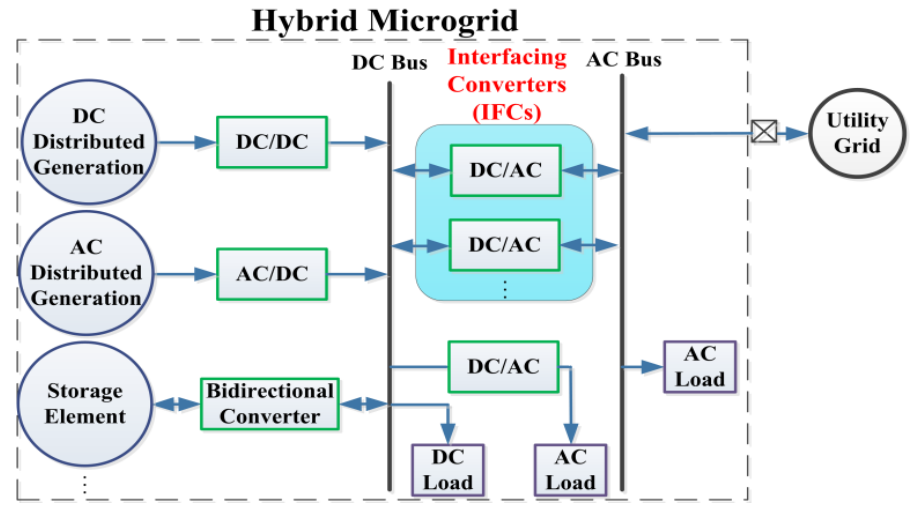

Figure. 3 DC-coupled hybrid microgrid

In DC-coupled hybrid MG, DC link voltage control, power balancing between generation and demand, and AC link voltage and frequency control (especially in stand-alone mode) are the important objectives of power management schemes. The overview of power management and control schemes of DCcoupled hybrid microgrid systems is shown in Figure 4. 


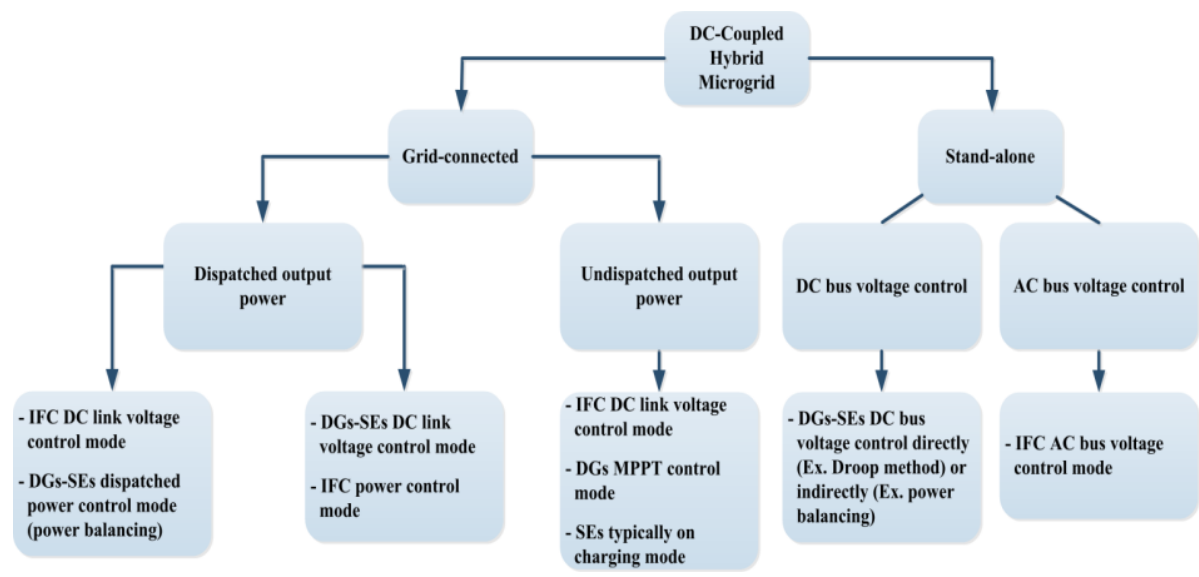

Figure 4. Power management strategies of the DC-coupled hybrid microgrid

In power control mode, converter output current or voltage is controlled to regulate IFC output power on its reference value. In DC link voltage control mode, IFC controls DC link voltage and balance the power generation and consumption on the DC bus. AC link voltage control mode of IFC is mainly for standalone microgrid operation, where IFC controls the AC subsystem voltage and frequency. For a gridconnected DC-coupled MG, if it is operated in dispatched power mode, DC link voltage can be controlled by two methods. In the first method, IFC works on DC link voltage control mode, and regulates DC link voltage at the desired value; DGs and SEs provide dispatched power of hybrid MG through power balancing control. By the second method, SEs on DC bus control, DC link voltage collectively using droop control method, and DGs can be part of the droop control or work in MPP. Here, IFC operates in power control mode and provides dispatched power to the grid. In undispatched output power operation mode, IFC operates in DC link voltage control mode. In the stand-alone operation of a DC-coupled hybrid MG, DC and AC bus voltages and frequency should be controlled simultaneously. For AC bus voltage and frequency control, IFC works on AC link voltage control mode and controls the AC bus voltage and frequency, whereas, DC bus voltage can be controlled directly or indirectly.

\subsection{AC-DC-coupled hybrid microgrid}

In AC-DC-coupled hybrid MG, as shown in Figure 5, multiple DGs and SEs are connected to DC and $\mathrm{AC}$ buses, linked by the interlinking converter (ILC) with minimized power conversion requirements.

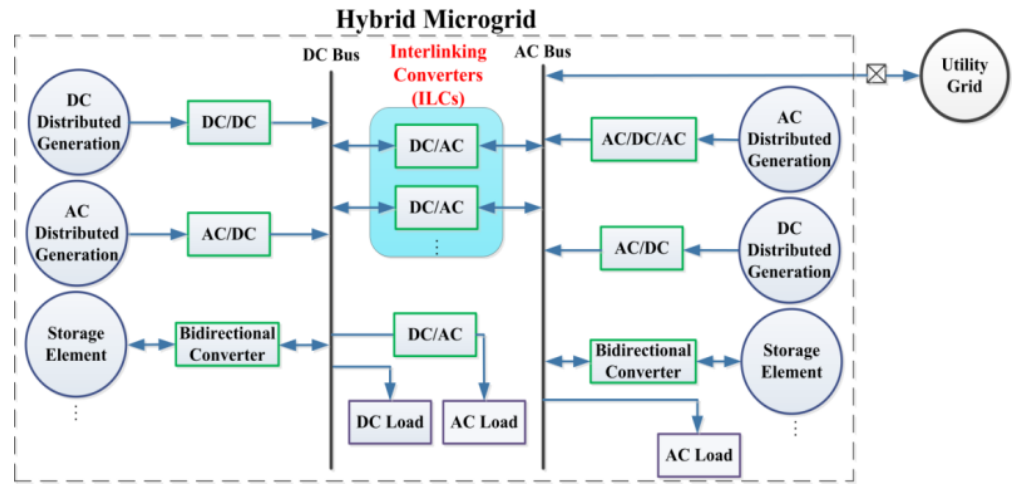

Figure 5. AC-DC-coupled hybrid microgrid

An overview of power management schemes of AC-DC-coupled hybrid MG is shown in Figure 6. The ILC in an AC-DC-coupled MG can be in bidirectional power control mode, DC voltage control mode, or AC voltage control mode. In grid-connected operation mode with dispatched MG output power, two methods can be used for DC-link voltage control and dispatched power generation. In the first method, ILC works on DC link voltage regulation mode to set the DC bus voltage on its desired value. In this mode, coordination 
between DGs-SEs on the DC bus and DGs-SEs on the AC bus is necessary to produce the dispatched output powers. In the second operation mode, DGs-SEs on the DC bus regulate DC link voltage on its reference value, while ILC and DGs-SEs on the AC bus collectively provide the dispatched power. In this operation mode, ILC works on power control mode.

In grid-connected undispatched output power operation mode, DGs in both DC and AC buses work on MPP. In this mode, ILC regulates DC link voltage on its desired value and injects all power generated by DGs-SEs in the DC bus to the load/grid.

In stand-alone operation mode, coordination among ILC, DGs-SEs on AC bus, and DGs-SEs on DC bus are essential to regulate $\mathrm{DC}$ bus voltage, $\mathrm{AC}$ bus voltage and frequency, and balance microgrid total generation and demand powers at the same time. In this operation mode, the PM strategies of AC-coupled hybrid MG in stand-alone operation mode such as droop and master-slave. can be used for AC subsystem voltage and frequency regulation and demand power-sharing. For the DC subsystem control, similar to DCcoupled hybrid MG in stand-alone operation, DC bus voltage can be controlled by DGs-SEs on DC bus directly.

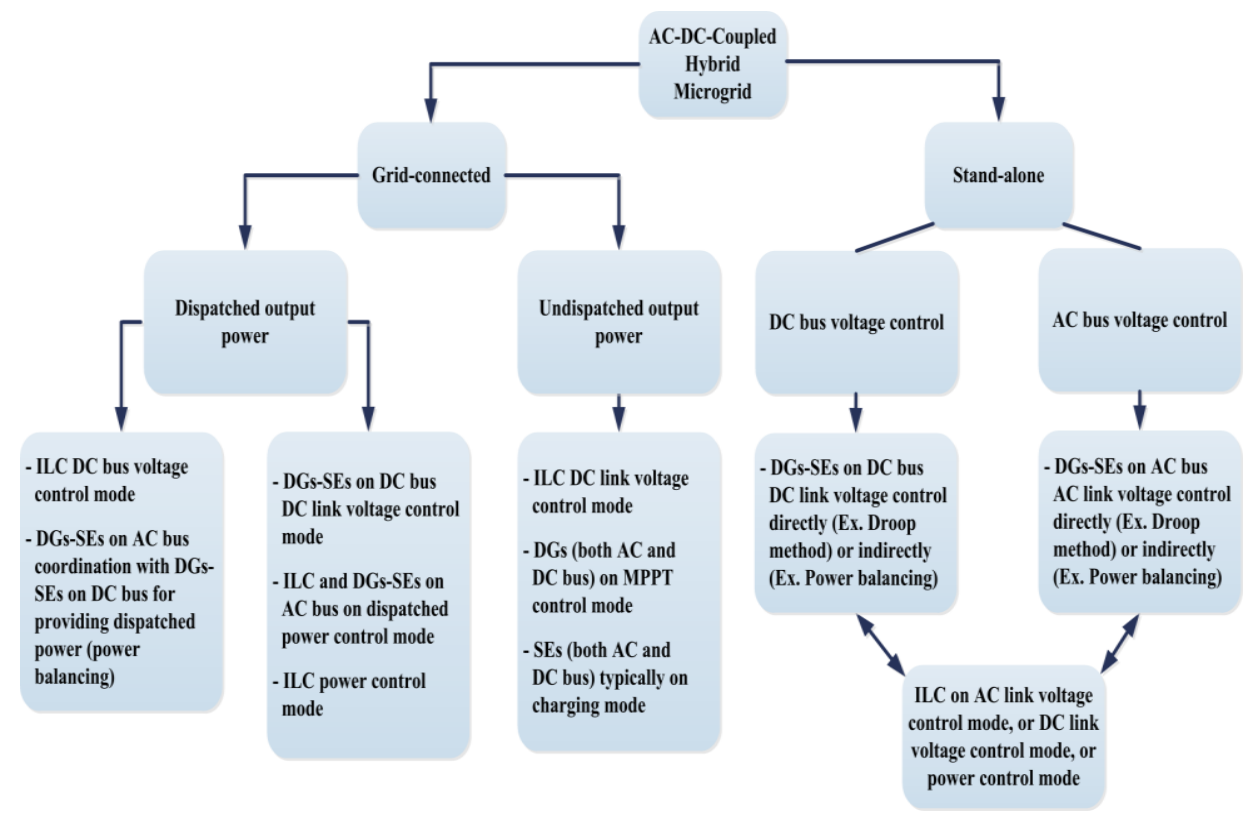

Figure 6. Power management strategies of the AC-DC-coupled hybrid microgrid

Depending on the types of control strategies used in AC and DC buses, this converter can be used on DC-bus control mode, AC-bus control mode, or output power control mode. DC bus voltage is controlled by DGs-SEs connected to DC bus, and AC bus voltage is controlled by DGs-SEs connected to AC bus, the ILC is responsible to manage the power flow between AC and DC sides to equalize the demand and generated power.

\section{CONTROL STRATEGIES EMPLOYED FOR MICROGRID OPERATION}

The microgrid normally operates in a centralized or decentralized model [5]. This section states some characteristics of control schemes employed for its operation as in Table 2.

Table 2. Properties of centralized/decentralized control

\begin{tabular}{lll}
\hline \multicolumn{1}{c}{ Criteria } & \multicolumn{1}{c}{ Centralized control } & \multicolumn{1}{c}{ Decentralized control } \\
\hline Mode of operation & Common goals & Market/competitive actions \\
Ownership & Not diverse & Diverse \&objective \\
Device operability & Follows central controller's command. & Independent and intelligent. \\
Number of DERs and loads & Limited & Normally high. \\
Dedicated communication & Available & Not available \\
Solution approach & Optimal & Suboptimal \\
\hline
\end{tabular}




\subsection{Primary control}

Primary control (PC)is the basic control level of an MG hierarchical control structure, which follows the operational command from upper levels and regulates the DG tie converters to realize their committed functions. PC is responsible for individual converter power, voltage, and frequency regulation. Droop control and virtual impedance are also used on top of inner voltage and current loops. A typical MG configuration with decentralized primary control is shown in Figure 7, consisting of renewable energy source (RES), energy storage system (ESS), and distributed loads. Here the common challenge is the management of uncertain RES generation and load consumption. So, RESs are usually operated at maximum power point (MPP). Hence, ESSs become indispensable components to achieve autonomous operation of MGs, while the limitation of ESS energy and power capacity requires a reasonable regulation strategy to prevent over-charge or over-discharge.

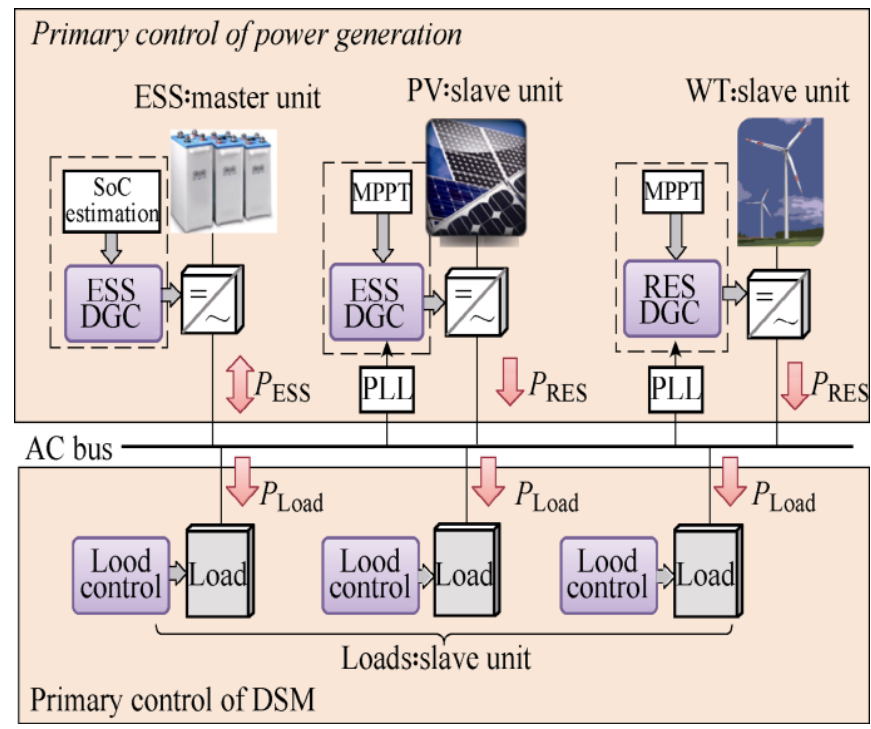

Figure 7. The decentralized primary control scheme

\subsection{Secondary control}

The main function of secondary control is to perform power quality regulations to manage voltage/frequency deviations, unbalances, and harmonics, which encompasses a synchronization loop between the MG and external grid.

\subsubsection{Centralized secondary control}

Figure 8 shows centralized secondary control architecture for MG consisting of some DGs controlled by local primary control and one central secondary controller, which collects remotely measured variables transferred utilizing a low bandwidth communication system.

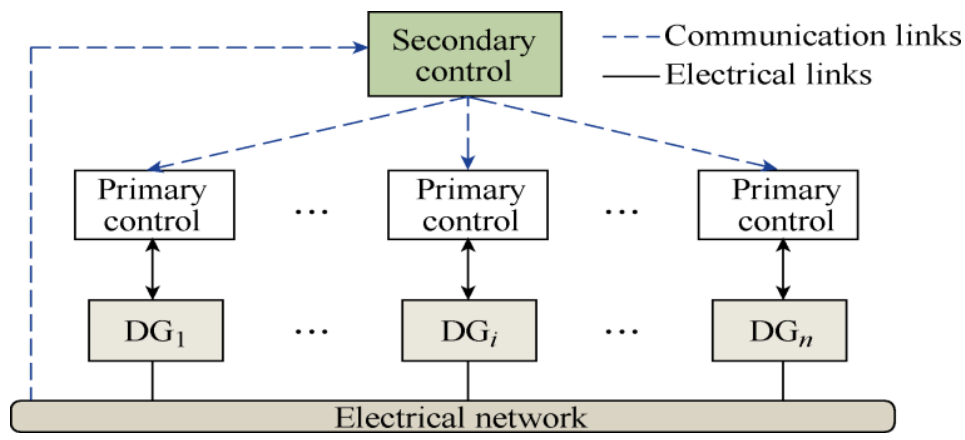

Figure 8. Scheme of centralized secondary control 


\subsubsection{Distributed secondary control}

Distributed secondary control (DSC) controls central controllers with less communication and computation costs while improving the reliability of the control system. Here the primary and secondary control operates together into one local controller. However, these local controllers need to "talk" with their companions, as shown in Figure 9.

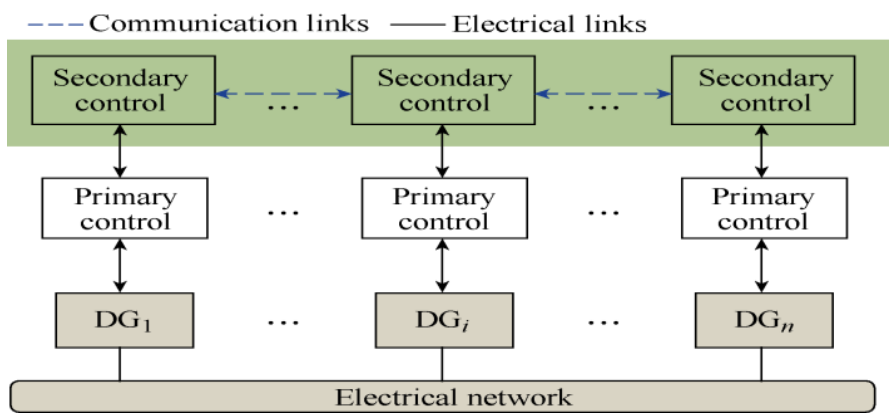

Figure 9. General scheme of distributed secondary control

DSC is used to exchange the information through the neighboring communication, by utilizing a distributed protocol. Its main function is to shift/change the droop characteristics of associated inverters to perform the restoration of voltage (and frequency) levels to nominal values or values that ensure proper power-sharing among DGs in the system.

\subsection{Tertiary control}

Tertiary control is in charge of regulating power exchange with external grid or/and with other MGs, which includes advanced functions related to efficiency and economic enhancements constituting a higher management level [6]-[11]. Tertiary control is located at the top level of an MG hierarchical control system, as shown in Figure 10. Figure 11 shows the control strategies employed for the microgrid.

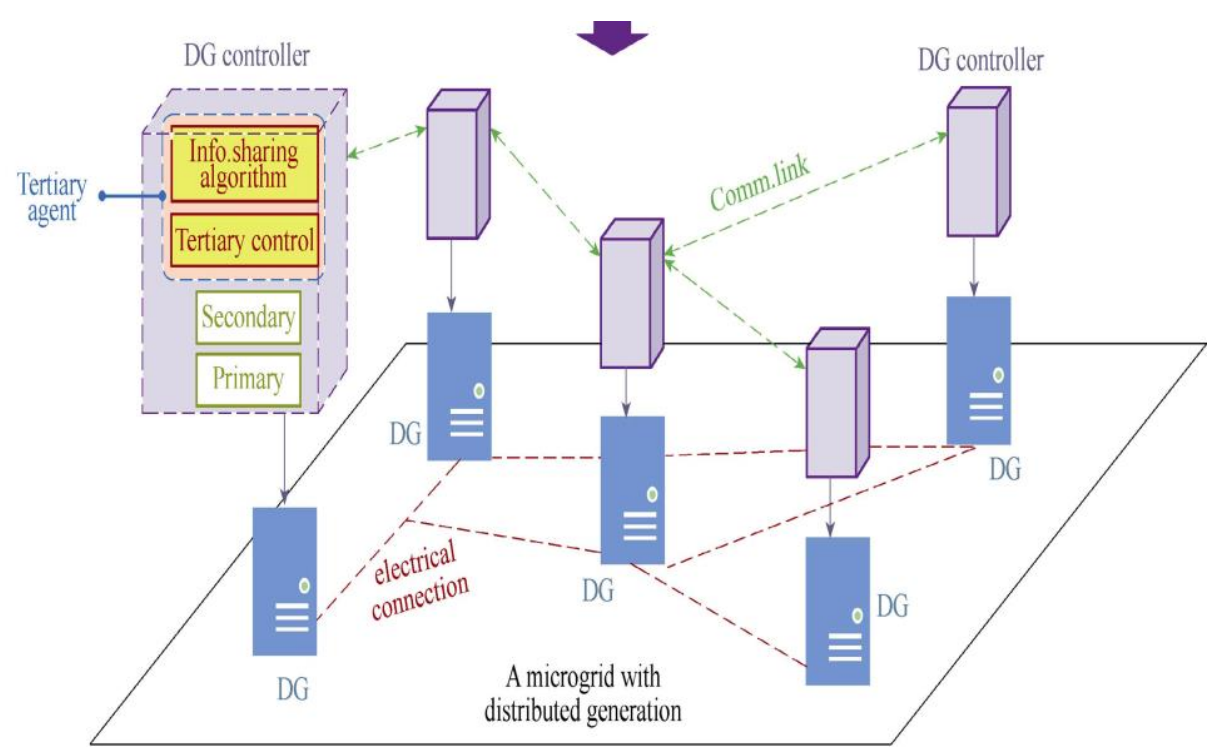

Figure 10. Distributed agent-based hierarchical control system for microgrids 


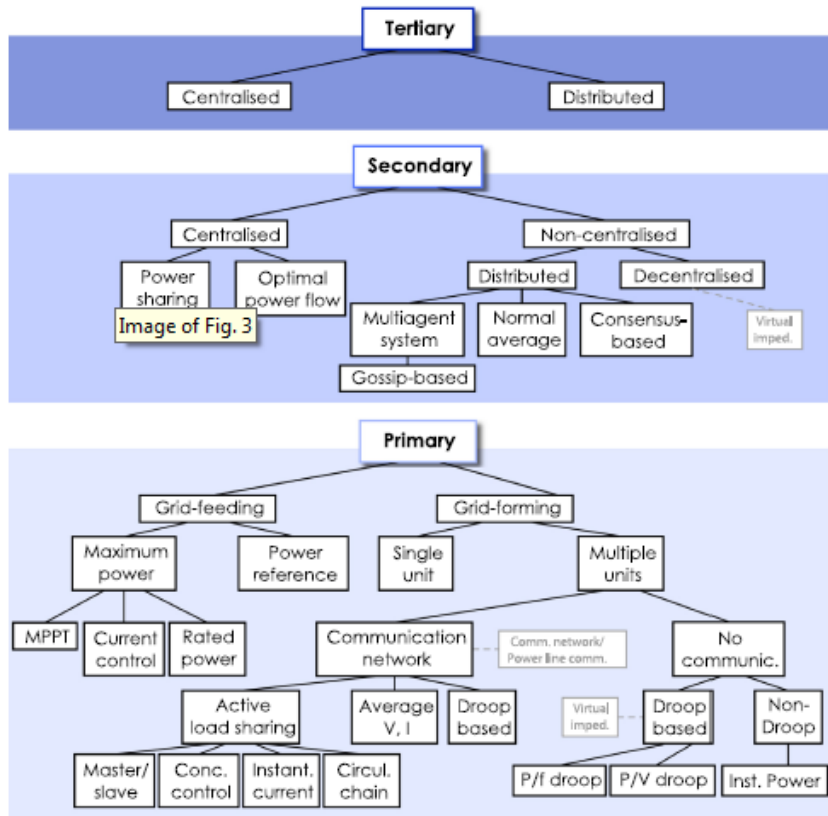

Figure 11. Microgrid control strategies

\section{PROTECTION SCHEMES DEVELOPED FOR MICROGRID OPERATION}

Micro-grid protection schemes can be classified into the following types such as adaptive protection, differential protection, distance protection, voltage-based protection, over current protection, techniques with external devices utilization, and coordinated protection techniques [12].

\subsection{Adaptive protection scheme}

This scheme is an online system that can modify the desired protective response to change under system conditions or requirements appropriately by the use of external control actions or generated signals by employing numerical directional overcurrent relays. Here, an automatic readjustment of relay settings occurs when MG changes from grid-connected mode to islanded mode and vice versa. For efficient protection, the standard communication protocol is needed to enable relays to communicate and exchange information with a central computer or between different relays.

\subsection{Differential protection scheme}

This scheme compares the currents entering and leaving a chosen protected zone and operates when the difference between these currents exceeds a predetermined magnitude.

\subsection{Distance protection scheme}

This scheme uses impedance measurements to effectively detect faults. It is able to isolate a fault that happens on either side of the protected circuit and also has the ability to operate in the case of reverse faults but reach settings, different for forward and reverse faults.

\subsection{Voltage-based protection scheme}

This scheme is used to protect power systems against numerous faults by measuring the voltages and protecting against both in and out-of-zone faults circumstances. A communication link is required in the scheme to categorize which faults are in-zone or out-of-zone.

\subsection{Coordinated protection technique}

Protection schemes should be coordinated to enhance efficiency in operations of primary and backup lines of defense. Primary protection schemes act as the first line of protection against faults, while backup protection schemes act in the event of primary protection failure. This process makes the protection scheme more resilient and reliable in operation.

Key factors in microgrid protection are based on type of microgrid and its topology, type of DG resources, communication type, time delay of communication links, method of analyzing data and detecting 
faults, fault type, and method of grounding. Different methods of protecting microgrids are changing protective devices or settings, disconnecting DG units during faults, creating a balance among different DG technologies, using fault current limiter/smart transformer and centralized/decentralized/adaptive protection schemes [13].

Challenges in microgrid protection are a) changes in the short-circuit level, b) false tripping, c) blindness of protection, d) prohibition of automatic reclosing, e) unsynchronized reclosing, f) dynamics in fault current magnitude, g) Loss of mains (LOM), and h) unnecessary disconnections [14].

Future trends in control strategies for islanded microgrid are essentially related to a) energy services: including demand response, optimal power flow, market participation, and storage management, b) microgrid protection especially in meshed topologies, c) performance optimization under the high-penetration level of DG resources, d) cost prioritized droop schemes, e) self-healing ability implementation, f) stability improvement in the cases of complex load including dynamic loads, constant loads, induction motor, pulsed loads, and electric vehicles, g) DG source dynamics, h) three-phase four-wire microgrids, i) cyber security improvement, j) reducing or eliminating the communication links, and k) studying DC and hybrid microgrids [15].

\section{DEVELOPMENTS IN DC MICROGRIDS}

Ministry of New and Renewable Energy (MNRE), Government of India had issued a draft policy for mini and microgrids for India in June 2016. This policy aims to increase microgrid capacity to 500 MW in the next five years in the private sector, which includes the deployment of roughly 10,000 renewable energybased mini and micro-scale projects averaging $50 \mathrm{~kW}$ across the country. Some highlights of the policy include a) regulated price determination for mini-grid projects (with tariff determination flexibility provided to operators), b) provision of single-window clearances for seeking right of way and regulatory approvals, and the availability of information on taxes, c) local village committee creation to ensure payment collection, customer adoption, and easier dispute resolution, d) grid connection provision to enable the sale of power to utilities, and e) renewable purchase obligation multiplier to make interconnections enabling attractive options for distribution companies, the specification of standards, performance, and quality. The following are the voltage and power levels are mentioned in the policy for DC/AC microgrids: For DC microgrids: a) 24V DC systems up to $1 \mathrm{kWp}$ capacity, and b) $72 \mathrm{~V} \mathrm{DC}$ systems for more than $1 \mathrm{kWp}$ and up to $10 \mathrm{kWp}$ capacity. For AC microgrids: a) $220 \mathrm{~V}$ single-phase systems up to $10 \mathrm{kWp}$ capacity, and b) $440 \mathrm{~V}$ three-phase AC systems for capacities beyond $10 \mathrm{kWp}$ capacity. Most of the successfully implemented microgrid projects employ mini-hydro, solar PV, biomass, and wind as a source of power generation.

The main benefits of using dc microgrids are a) significant energy savings due to reduced number of energy conversion (AC to DC and vice versa) processes, and b) savings of between 20 and $50 \%$ of electricity with dc electrical loads, such as LED lights, fans with brushless dc motors, and other DC-powered electronics, compared to ac powered appliances, such as compact fluorescent lamp (CFL) lighting and induction motors connected with ac adapters. Few solar-powered $48 \mathrm{~V}$ DC power distribution system/DC microgrid has been implemented in school/rooftop apartments in India [16]-[18].

A general structure of DC microgrids is shown in Figure 12(a). In DC microgrids, three-phase ACto-DC rectifiers and transformers are required to connect AC DERs to the common bus, single and threephase DC-to-AC inverters are needed for supplying AC loads, and a three-phase DC-to-AC/AC-to-DC converter, a transformer, and a point of common coupling switch are required for connecting the microgrid to the utility grid. In this figure, the direction of arrows shows the direction of power flow. Also, different DC loads require different DC voltage levels, so DC-to-AC converters have to be considered as well to change the voltage level of the DC sources to desired levels. A common DC bus can represent one or more loop/radial distribution networks that connect loads and DERs within the microgrid to handle DC voltages and currents. In a DC MG system, the energy sources and power electronic loads can be supplied more effectively and efficiently by choosing a suitable voltage level and thereby avoiding a few conversion stages as shown in Figure 12(b). Furthermore, the ESS can be directly connected to the main DC bus or connected via a DC-DC converter [19]-[21].

DC microgrids could offer several advantages when compared with AC microgrids such as a) higher efficiency and reduced losses due to the reduction of multiple converters used for DC loads, b) easier integration of various DC DERs, such as energy storage, solar PV, and fuel cells, to the common bus with simplified interfaces, c) more efficient supply of DC loads, such as electric vehicles and LED lights, d) eliminating the need for synchronizing generators, which enables rotary generating units to operate at their optimum speed, and e) enabling bus ties to be operated without the need for synchronizing the buses [22]-[24]. 


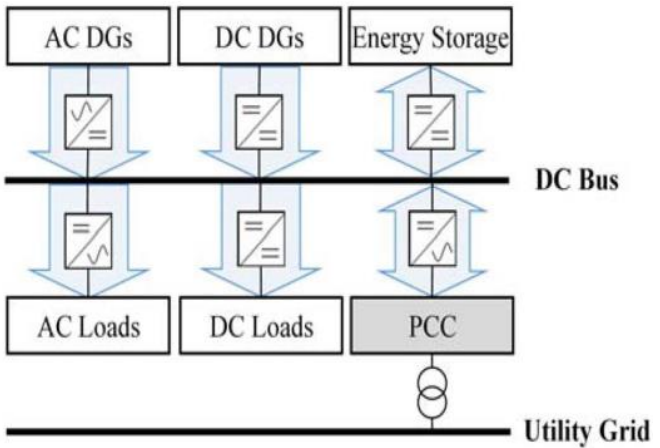

(a)

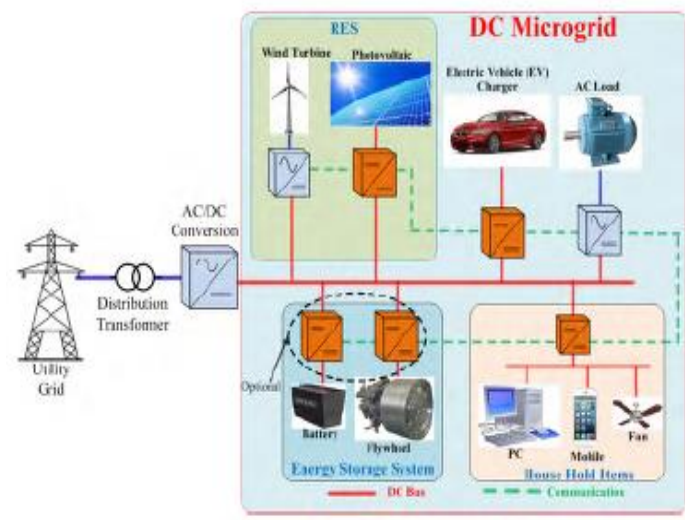

(b)

Figure 12. DC microgrids: (a) general structure of DC microgrids and (b) building block of DC microgrids

Salomonsson et al. [25] describe the framework for the expansion planning of off-grid microgrids. As shown in Figure 13, the long-term expansion planning studies are composed of three major components such as data collection and synthesization, long-term expansion planning and short-term operation planning.

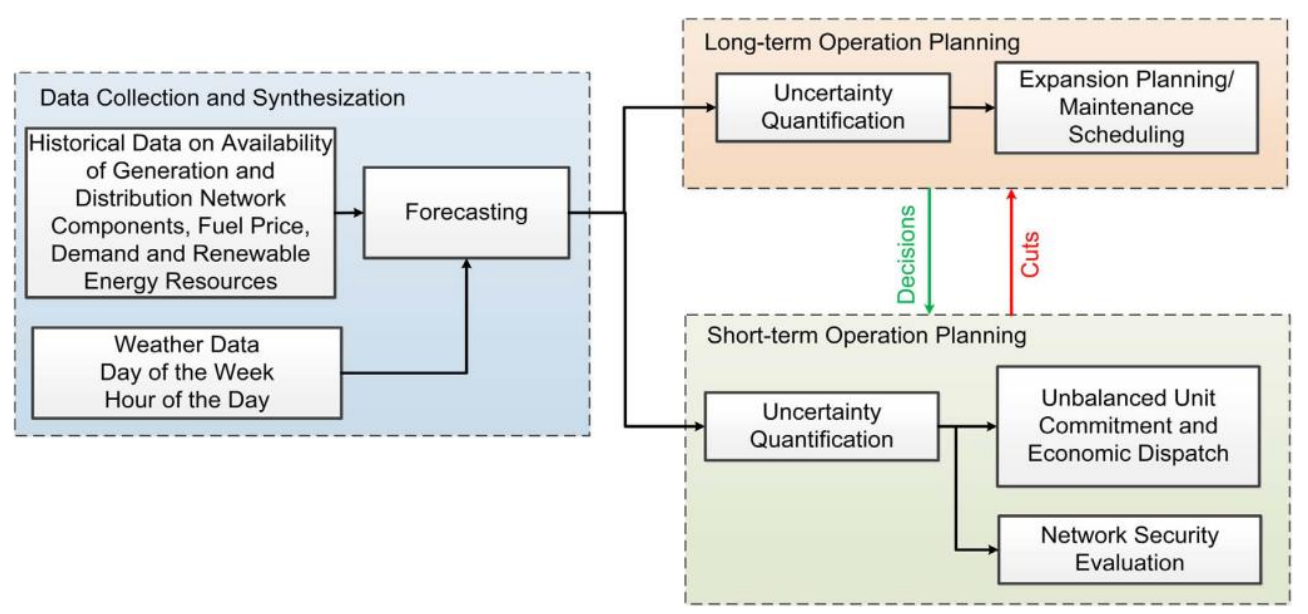

Figure 13. Framework for the long-term expansion planning of off-grid microgrids

\section{TECHNO ECONOMICAL ASPECTS, STANDARDS OF DC MICROGRID}

DC microgrid offer several benefits such as a) higher efficiency and reduced losses due to the reduced number of multiple converters, b) easier integration of various dc DERs to the common bus with simplified interfaces, c) efficient supply of DC loads, such as electric vehicles and LED lights, d) simple models and controls (i.e., no phase angle or frequency or reactive power), without the need for synchronizing the buses, e) higher compatibility due to same nature of sources and emerging loads, f) improved system reliability, efficiency, and economy by eliminating the DC/AC/DC conversion stages, g) absence of skin effect, h) safer for human bodies, since discharges are lower than AC, and i) high power transfer capacity [26]-[30].

DC microgrids can be economical in following cases: a) when the ratio of dc loads is high, b) installation with PV-based power generation as its generation pattern matches with the market price and load variations, and c) suitable for critical loads. Application of DC microgrids are a) commercial and residential building, b) industrial systems, c) data centers, d) telecommunication systems, e) electric vehicle fast charging stations, and f) traction/ship/marine/aircraft systems.

DC micro grid with hybrid power generation and energy storage is the simplest, reliable, cost effective, scalable, and highly efficient solution to provide access to electricity to people living without access to electricity. For successful implementation of DC microgrids project, proper planning and 
development, effective construction, reliable energy generation and power delivery, best operation and maintenance activities including constant plant monitoring and control, preventive maintenance, fault detection and response and corrective action are needed [31]-[37]. Some of the DC microgrid protection challenges are a) direction of fault current, b) coordination problem of current-based relay, c) non-suitability of AC circuit breakers, d) change in the SC level, e) low fault current capacity of inverters, and f) grounding. DC microgrids use PDs such as fuses, relays, and actuators such as DC CBs, switches and breaker less.

Obstacles in the practical implementation of the DC microgrid, are a) no natural zero crossing of the current in DC grid make it difficult, b) transition from AC to DC system in low voltage distribution networks requires several stages such as new standards for products and voltage levels, c) grounding and corrosion issues in DC systems. The major issues and potential solutions in microgrid protection and control include bidirectional power flows, short circuit capacity, stability issues, low inertia, and intermittent output. Major risks of mini-grid operations are a) early recognition of crucial risk driving factors, b) maintaining a balance between chances and risks, c) initiating focused actions at an early stage to limit risk exposure, and d) opening up options for new projects. Risk management measures to overcome major risks are a) political risks avoided by insurance instruments, b) risk of non-payment, and c) risk of resource price variability. The most common power quality issues in DC microgrid systems are voltage transient from AC grid, harmonics due to resonances and power electronics-based converters, electromagnetic interference and compatibility issues, communication failures, inrush currents, DC bus faults, voltage unbalance in bipolar DC bus and circulating currents. The available DC microgrid standards are listed in the following Table 3.

Table 3. DC microgrid standards

\begin{tabular}{|c|c|c|}
\hline Sl. No. & Standards & Specifications \\
\hline 1 & International Electrotechnical Commission & IEC 62040-5-3, IEC 61643-3 and IEC 61643-311 \\
\hline 2 & $\begin{array}{l}\text { The Institute of Electrical and Electronic Engineering } \\
\text { Standard Association (IEEE-SA) }\end{array}$ & WG946, P2030.10, IEEE DC@Home \\
\hline 3 & Emerge Alliance & $\begin{array}{l}\text { EMerge Alliance Occupied Space Standard, EMerge Alliance } \\
\text { Data/Telecom Center Standard }\end{array}$ \\
\hline 4 & European Telecom Standard Institute (ETSI) & ETSI EN 300 132-3-1 \\
\hline 5 & International Telecommunication Union (ITU) & ITU L.1201/1202/1203 \\
\hline 6 & Chinese Communication Standards Association (CCSA) & YD/T2378-2011, YD/T 3091-2016 \\
\hline
\end{tabular}

Microgrids are needed to address ramping, frequency/voltage control, non-spinning substitute for spinning reserve and overall power quality for end use customers. MG provides resiliency, sustainability and commercial viability and provides value to both the local utility and the customer. Figure 14 shows the value chain of microgrid as a service.

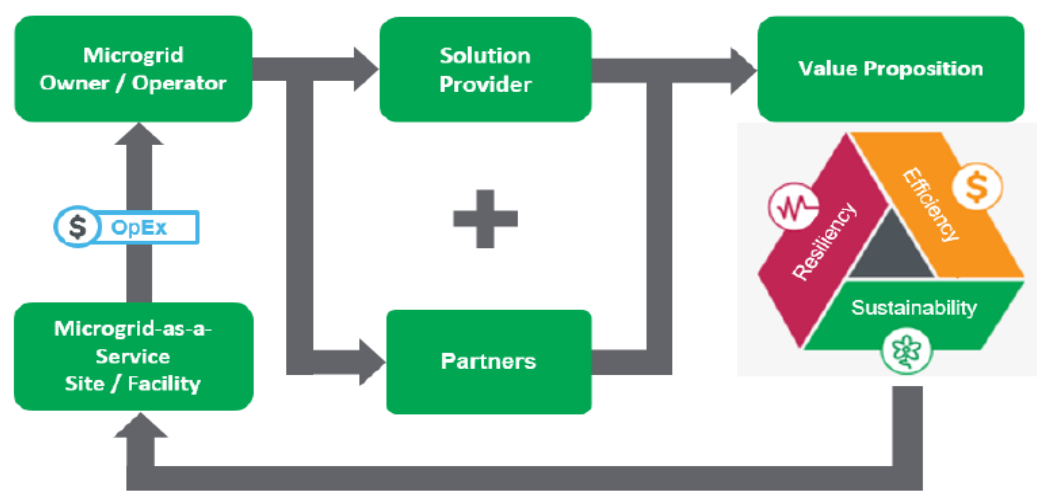

Figure 14. Value chain of microgrid as a service

\section{PERFORMANCE EVALUATION APPROACHES OF MICROGRID}

Pretea et al. [38] have made sustainability and reliability assessment of microgrids in the regional electricity market through various indicators such as environmental, economic and technical factors, which are briefed as environmental indicators are: a) annual emissions of COx (Mton/yr), b) annual emissions of NOx (kton/yr), c) annual emissions of SOx (kton/yr); Economic indicators are: a) annualized capital costs 
and variable costs (INR/yr), and b) annualized capital costs and variable costs, including environmental externalities (INR/yr); Technical indicators are: a) annual energetic electric efficiency of the network, b) annual energetic total efficiency of the network, c) annual exergetic electric efficiency of the network, and d) annual exergetic total efficiency of the network. The two reliability indicators are: a) annual loss of load probability (outage hours/10 years), and b) annual expected loss of energy (MWh/year).

He et al. [39] have quantified methods for vulnerability pre-assessment of microgrid through two indices such as power generation capacity adequacy and power supply vulnerability. Generation capacity adequacy refers to the capability of the MG able to meet the electric power requirement of users, keeping the system conditions within the allowable range and power supply vulnerability mainly depends on the five indices, such as load/overload/overvoltage/low load power factor/three-phase unbalance risks.

Ma et al. [40] have listed assessment indices for reliability evaluation of microgrid as given in Table 4 such as microgrid system important load average interruption frequency index (MSILAIFI), microgrid system important load average interruption duration index (MSILAIDI), microgrid system important load average service availability index (MSILASAI) and microgrid system important load energy not service index (MSILENSI), by assuming that the total number of the load points in a certain microgrid is $\eta$, total number of customers of the load point $\mathrm{i}$ is $\mathrm{N}_{\mathrm{i}}$, important customer weight of this load point $\mathrm{i}$ is $\beta_{\mathrm{i}}, \mathrm{U}_{\mathrm{i}}$ is the average annual outage duration of point load $\mathrm{I}, \mathrm{L}_{\mathrm{ai}}$ is the average load connected to the power outage load point $I$ and then the total number of important customers of load point i is $\mathrm{N}_{\mathrm{i}} \beta_{\mathrm{i}}$.

Table 4. Reliability evaluation indices

\begin{tabular}{ccc}
\hline Sl. No. & Indices & Formula \\
\hline 1 & MSILAIFI & $\frac{\sum_{i}^{n} \lambda_{i} N_{i} \beta_{i}}{\sum_{i=1}^{n} N_{i} \beta_{i}}$ (outage/customer year) \\
2 & MSILAIDI & $\frac{\sum_{i}^{n} U_{i} N_{i} \beta_{i}}{\sum_{i=1}^{n} N_{i} \beta_{i}}$ (hours/customer year) \\
3 & MSILASAI & MSILAIDI \\
4 & MSILENSI & \multirow{2}{*}{$\sum_{i=1}^{n}$ LaiU $_{i} \beta_{i}$ (kWh $/$ year) } \\
& & \multicolumn{2}{c}{} \\
\hline
\end{tabular}

Compositional power flow [41] is devised for networked microgrids to account power sharing and voltage regulation between microgrids while preserving data privacy of each microgrid. The main contributions of this approach include: a) devising an advanced-droop-control based power flow to incorporate distributed energy resources and load droops within microgrids, and b) establishing an adaptivesecondary-control-based compositional power flow scheme to account for power sharing and voltage regulation between microgrids. ComPF supports plug-and-play of microgrids and preserves customer privacy.

Microgrid robust economic viability assessment under lasting uncertainty enclosure (MGREVALUE) [42] is a robust optimization tool capable of returning the optimal microgrid sizing and configuration for each unique microgrid deployment scenario. This tool provides a viable means of simulation and optimization for microgrid deployments based on selected user inputs which includes: DERs considered by the user, minimum and maximum load capacity, current and future energy consumption forecasts, desired reliability performance levels, budget constraints, and the uncertainty thresholds. The tool gathers this information via a graphical user interface (GUI) and converts it into an acceptable format that can be given to a CPLEX® core optimization engine for analysis. For a given objective, this tool produces the optimal generation mix to be utilized in the microgrid, the investment payback period, total and annual investment and operation costs, and potential total and annual revenues as a result of the DER installation, amassing to the overall economic viability for a specific microgrid deployment.

\section{CONCLUSION}

The microgrid has the attributes like higher reliability, improved power quality, reduced emissions, reduced network congestion/power losses, increased energy efficiency, natural interface with RESs, electronic loads and ESSs. This paper addresses the operation aspects of microgrids to eliminate investments on additional generation and transmission facilities to supply remote loads. Moreover, microgrid's islanding capability in the event of faults or disturbances in upstream networks would enhance grid and customers' reliability and resilience are addressed through the discussion of various control and protection schemes and reliability indices. 


\section{ACKNOWLEDGMENT}

This work is supported by Indo-Sri Lanka Joint Research Program by Department of Science \& Technology(DST), Government of India and Ministry of Science, Technology \& Research (MSTR), Government of Sri Lanka, through grant in aidsDST:14.00.31.14.60.798.60.3425 and MSTR/TR/AGR/3/02/13 respectively.

\section{REFERENCES}

[1] S. Parhizi, H. Lotfi, A. Khodaei, and S. Bahramirad, "State of the art in research on microgrids: A review," IEEE Access, vol. 3, pp. 890-925, 2015, doi: 10.1109/ACCESS.2015.2443119.

[2] H. Lotfi and A. Khodaei, "AC versus DC microgrid planning," IEEE Transactions on Smart Grid, vol. 8, no. 1, pp. 296-304, Jan. 2017, doi: 10.1109/TSG.2015.2457910.

[3] M. Nasir, H. A. Khan, A. Hussain, L. Mateen, and N. A. Zaffar, "Solar PV-based scalable DC microgrid for rural electrification in developing regions," IEEE Transactions on Sustainable Energy, vol. 9, no. 1, pp. 390-399, Jan. 2018, doi: 10.1109/TSTE.2017.2736160.

[4] F. Nejabatkhah and Y. W. Li, "Overview of Power Management Strategies of Hybrid AC/DC Microgrid," IEEE Transactions on Power Electronics, vol. 30, no. 12, pp. 7072-7089, Dec. 2015, doi: 10.1109/TPEL.2014.2384999.

[5] T. Dragicevic, D. Wu, Q. Shafiee, and L. Meng, "Distributed and decentralized control architectures for converterinterfaced microgrids," Chinese Journal of Electrical Engineering, vol. 3, no. 2, pp. 41-52, Sep. 2017, doi: 10.23919/CJEE.2017.8048411.

[6] L. Chen and S. Mei, "An integrated control and protection system for photovoltaic microgrids," CSEE Journal of Power and Energy Systems, vol. 1, no. 1, pp. 36-42, Mar. 2015, doi: 10.17775/cseejpes.2015.00005.

[7] S. A. Alavi, K. Mehran, Y. Hao, A. Rahimian, H. Mirsaeedi, and V. Vahidinasab, "A distributed event-triggered control strategy for dc microgrids based on publish-subscribe model over industrial wireless sensor networks," IEEE Transactions on Smart Grid, vol. 10, no. 4, pp. 4323-4337, Jul. 2019, doi: 10.1109/TSG.2018.2856893.

[8] D. Yang, Z. Xu, W. Li, and F. Bu, "Droop control strategy of the AC/DC hybrid micro-grid based on quasi-PR control," The Journal of Engineering, vol. 2017, no. 14, pp. 2634-2642, Jan. 2017, doi: 10.1049/joe.2017.0840.

[9] M. Khederzadeh, "Multi-agent system design for automation of a cluster of microgrids," CIRED - Open Access Proceedings Journal, vol. 2017, no. 1, pp. 1304-1307, Oct. 2017, doi: 10.1049/oap-cired.2017.0074.

[10] M. Starke, A. Herron, D. King, and Y. Xue, "Implementation of a Publish-Subscribe Protocol in Microgrid Islanding and Resynchronization with Self-Discovery," IEEE Transactions on Smart Grid, vol. 10, no. 1, pp. 361370, Jan. 2018, doi: 10.1109/TSG.2017.2739246.

[11] W. Sun, S. Ma, I. Alvarez-Fernandez, R. R. Nejad, and A. Golshani, "Optimal self-healing strategy for microgrid islanding," IET Smart Grid, vol. 1, no. 4, pp. 143-150, Dec. 2018, doi: 10.1049/iet-stg.2018.0057.

[12] X. Kang, C. E. K. Nuworklo, B. S. Tekpeti, and M. Kheshti, "Protection of micro-grid systems: a comprehensive survey,” The Journal of Engineering, vol. 2017, no. 13, pp. 1515-1518, Jan. 2017, doi: 10.1049/joe.2017.0584.

[13] S. A. Hosseini, H. A. Abyaneh, S. H. H. Sadeghi, F. Razavi, and A. Nasiri, "An overview of microgrid protection methods and the factors involved," Renewable and Sustainable Energy Reviews, vol. 64, pp. 174-186, Oct. 2016, doi: 10.1016/j.rser.2016.05.089.

[14] B. J. Brearley and R. R. Prabu, "A review on issues and approaches for microgrid protection," Renewable and Sustainable Energy Reviews, vol. 67, pp. 988-997, Jan. 2017, doi: 10.1016/j.rser.2016.09.047.

[15] M. H. Andishgar, E. Gholipour, and R. allah Hooshmand, "An overview of control approaches of inverter-based microgrids in islanding mode of operation," Renewable and Sustainable Energy Reviews, vol. 80, pp. 1043-1060, Dec. 2017, doi: 10.1016/j.rser.2017.05.267.

[16] V. A. Suryad, S. Doolla, and M. Chandorkar, "Microgrids in India: Possibilities and challenges," IEEE Electrification Magazine, vol. 5, no. 2, pp. 47-55, Jun. 2017, doi: 10.1109/MELE.2017.2685880.

[17] K. Shenai, A. Jhunjhunwala, and P. Kaur, "Electrifying India: Using solar dc microgrids," IEEE Power Electronics Magazine, vol. 3, no. 4, pp. 42-48, Dec. 2016, doi: 10.1109/MPEL.2016.2614905.

[18] A. Jhunjhunwala, A. Lolla, and P. Kaur, "Solar-dc Microgrid for Indian Homes: A Transforming Power Scenario," IEEE Electrification Magazine, vol. 4, no. 2, pp. 10-19, Jun. 2016, doi: 10.1109/MELE.2016.2543950.

[19] P. A. Madduri, J. Poon, J. Rosa, M. Podolsky, E. A. Brewer, and S. R. Sanders, "Scalable DC Microgrids for Rural Electrification in Emerging Regions," IEEE Journal of Emerging and Selected Topics in Power Electronics, vol. 4, no. 4, pp. 1195-1205, Dec. 2016, doi: 10.1109/JESTPE.2016.2570229.

[20] K. T. Tan, B. Sivaneasan, X. Y. Peng, and P. L. So, "Control and Operation of a DC Grid-Based Wind Power Generation System in a Microgrid," IEEE Transactions on Energy Conversion, vol. 31, no. 2, pp. 496-505, Jun. 2016, doi: 10.1109/TEC.2015.2497709.

[21] Q. Ye, R. Mo, and H. Li, "Low-Frequency Resonance Suppression of a Dual-Active-Bridge DC/DC converter Enabled DC Microgrid," IEEE Journal of Emerging and Selected Topics in Power Electronics, vol. 5, no. 3, pp. 982-994, Sep. 2017, doi: 10.1109/JESTPE.2017.2700258.

[22] J. Won, W. Chae, H. Lee, J. Park, J. Sim, and C. Shin, "Demonstration of remote microgrid system in Korean island," CIRED - Open Access Proceedings Journal, vol. 2017, no. 1, pp. 2212-2214, Oct. 2017, doi: 10.1049/oapcired.2017.0683

[23] K. W. Joung et al., "Energy management system for stable operation of isolated microgrid," CIRED - Open Access Proceedings Journal, vol. 2017, no. 1, pp. 1737-1740, Oct. 2017, doi: 10.1049/oap-cired.2017.0288. 
[24] B. Cornélusse, D. Ernst, L. Warichet, and W. Legros, "Efficient management of a connected microgrid in Belgium," CIRED - Open Access Proceedings Journal, vol. 2017, no. 1, pp. 1729-1732, Oct. 2017, doi: 10.1049/oap-cired.2017.0211.

[25] D. Salomonsson, L. Söder, and A. Sannino, "Protection of low-voltage DC microgrids," IEEE Transactions on Power Delivery, vol. 24, no. 3, pp. 1045-1053, Jul. 2009, doi: 10.1109/TPWRD.2009.2016622.

[26] J. M. Guerrero, M. Chandorkar, T. L. Lee, and P. C. Loh, "Advanced control architectures for intelligent microgridspart i: Decentralized and hierarchical control," IEEE Transactions on Industrial Electronics, vol. 60, no. 4, pp. 1254-1262, Apr. 2013, doi: 10.1109/TIE.2012.2194969.

[27] J. M. Guerrero, P. C. Loh, T. L. Lee, and M. Chandorkar, "Advanced control architectures for intelligent microgridsPart II: Power quality, energy storage, and AC/DC microgrids," IEEE Transactions on Industrial Electronics, vol. 60, no. 4, pp. 1263-1270, Apr. 2013, doi: 10.1109/TIE.2012.2196889.

[28] M. Kumar, S. C. Srivastava, and S. N. Singh, "Control Strategies of a DC Microgrid for Grid Connected and Islanded Operations," IEEE Transactions on Smart Grid, vol. 6, no. 4, pp. 1588-1601, Jul. 2015, doi: 10.1109/TSG.2015.2394490.

[29] L. Meng et al., "Review on Control of DC Microgrids and Multiple Microgrid Clusters," IEEE Journal of Emerging and Selected Topics in Power Electronics, vol. 5, no. 3, pp. 928-948, 2017, doi: 10.1109/JESTPE.2017.2690219.

[30] A. Maknouninejad, Z. Qu, F. L. Lewis, and A. Davoudi, "Optimal, nonlinear, and distributed designs of droop controls for DC microgrids," IEEE Transactions on Smart Grid, vol. 5, no. 5, pp. 2508-2516, Sep. 2014, doi: 10.1109/TSG.2014.2325855.

[31] "DC Micro grid | Schneider Electric." https://www.se.com/in/en/product-range/62210-dc-micro-grid\#overview.

[32] N. Bayati, A. Hajizadeh, and M. Soltani, "Protection in DC microgrids: A comparative review," IET Smart Grid, vol. 1, no. 3, pp. 66-75, Oct. 2018, doi: 10.1049/iet-stg.2018.0035.

[33] K. Strunz, E. Abbasi, and D. N. Huu, "DC microgrid for wind and solar power integration," IEEE Journal of Emerging and Selected Topics in Power Electronics, vol. 2, no. 1, pp. 115-126, Mar. 2014, doi: 10.1109/JESTPE.2013.2294738.

[34] A. Werth, N. Kitamura, and K. Tanaka, "Conceptual Study for Open Energy Systems: Distributed Energy Network Using Interconnected DC Nanogrids," IEEE Transactions on Smart Grid, vol. 6, no. 4, pp. 1621-1630, Jul. 2015, doi: 10.1109/TSG.2015.2408603.

[35] T. Dragicevic, X. Lu, J. C. Vasquez, and J. M. Guerrero, "DC Microgrids - Part I: A Review of Control Strategies and Stabilization Techniques," IEEE Transactions on Power Electronics, vol. 31, no. 7, pp. 4876-4891, 2016, doi: 10.1109/TPEL.2015.2478859.

[36] T. Dragičević, X. Lu, J. C. Vasquez, and J. M. Guerrero, "DC Microgrids - Part II: A Review of Power Architectures, Applications, and Standardization Issues," IEEE Transactions on Power Electronics, vol. 31, no. 5, pp. 3528-3549, May 2016, doi: 10.1109/TPEL.2015.2464277.

[37] M. E. Khodayar, "Rural electrification and expansion planning of off-grid microgrids," Electricity Journal, vol. 30, no. 4, pp. 68-74, May 2017, doi: 10.1016/j.tej.2017.04.004.

[38] C. Lo Prete et al., "Sustainability and reliability assessment of microgrids in a regional electricity market," Energy, vol. 41, no. 1, pp. 192-202, May 2012, doi: 10.1016/j.energy.2011.08.028.

[39] W. He, G. Hua, H. Zheng, W. Fang, and H. Liu, "Quantitative method to pre-assess vulnerability for microgrid based on probability theory," The Journal of Engineering, vol. 2017, no. 13, pp. 1113-1117, Jan. 2017, doi: 10.1049/joe.2017.0502.

[40] T. Ma, J. Wu, and X. Niu, "Reliability assessment indices and method for urban microgrid," CIRED - Open Access Proceedings Journal, vol. 2017, no. 1, pp. 837-840, Oct. 2017, doi: 10.1049/oap-cired.2017.0693.

[41] Y. Li, P. Zhang, and C. Kang, "Compositional Power Flow for Networked Microgrids," IEEE Power and Energy Technology Systems Journal, vol. 6, no. 1, pp. 81-84, Mar. 2019, doi: 10.1109/jpets.2019.2897780.

[42] A. Khodaei, S. Bahramirad, E. A. Paaso, and M. Avendano, "Microgrid Economic Viability Assessment: An introduction to MG-REVALUE," Electricity Journal, vol. 30, no. 4, pp. 7-11, May 2017, doi: 10.1016/j.tej.2017.03.009. 